\title{
Assembly and proteolytic processing of mycobacterial ClpP1 and ClpP2
}

\author{
Nadia Benaroudj ${ }^{*}$, Bertrand Raynal ${ }^{2}$, Marika Miot ${ }^{3}$ and Miguel Ortiz-Lombardia ${ }^{4}$
}

\begin{abstract}
Background: Caseinolytic proteases (ClpPs) are barrel-shaped self-compartmentalized peptidases involved in eliminating damaged or short-lived regulatory proteins. The Mycobacterium tuberculosis (MTB) genome contains two genes coding for putative ClpPs, ClpP1 and ClpP2 respectively, that are likely to play a role in the virulence of the bacterium.

Results: We report the first biochemical characterization of ClpP1 and ClpP2 peptidases from MTB. Both proteins were produced and purified in Escherichia coli. Use of fluorogenic model peptides of diverse specificities failed to show peptidase activity with recombinant mycobacterial ClpP1 or ClpP2. However, we found that ClpP1 had a proteolytic activity responsible for its own cleavage after the Arg8 residue and cleavage of ClpP2 after the Ala12 residue. In addition, we showed that the absence of any peptidase activity toward model peptides was not due to an obstruction of the entry pore by the N-terminal flexible extremity of the proteins, nor to an absolute requirement for the ClpX or ClpC ATPase complex. Finally, we also found that removing the putative propeptides of ClpP1 and ClpP2 did not result in cleavage of model peptides.

We have also shown that recombinant ClpP1 and ClpP2 do not assemble in the conventional functional tetradecameric form but in lower order oligomeric species ranging from monomers to heptamers. The concomitant presence of both ClpP1 and ClpP2 did not result in tetradecameric assembly. Deleting the aminoterminal extremity of ClpP1 and ClpP2 (the putative propeptide or entry gate) promoted the assembly in higher order oligomeric species, suggesting that the flexible $\mathrm{N}$-terminal extremity of mycobacterial ClpPs participated in the destabilization of interaction between heptamers.

Conclusion: Despite the conservation of a Ser protease catalytic triad in their primary sequences, mycobacterial ClpP1 and ClpP2 do not have conventional peptidase activity toward peptide models and display an unusual mechanism of self-assembly. Therefore, the mechanism underlying their peptidase and proteolytic activities might differ from that of other ClpP proteolytic complexes.
\end{abstract}

\section{Background}

In all organisms, ATP-dependent proteases play an essential role by removing short-lived regulatory proteins whose rapid elimination is critical for cell metabolism and growth [1]. They also allow riddance of misfolded and damaged proteins that accumulate in a variety of circumstances, notably during environmental stress [2]. Simultaneous orchestrated action of key regulatory proteins as well as protein quality control

\footnotetext{
* Correspondence: nadia.benaroudj@pasteur.fr

'Institut Pasteur, Unité de Biologie des Spirochètes, Institut Pasteur, F-75015 Paris France

Full list of author information is available at the end of the article
}

mechanism assure successful survival and virulence of bacteria.

The Clp (caseinolytic protease) proteolytic system is one of the major ATP-dependent proteolytic complexes in bacteria. It consists of a barrel-shaped tetradecameric $\mathrm{ClpP}$ peptidase organized in two-stacked heptameric rings. The active sites for peptide bond cleavage are sequestered inside a proteolytic chamber accessible only through narrow axial pores and whose entry is restricted to unfolded polypeptides [3]. In E.coli, where the biochemical properties of ClpP have been extensively studied, the peptidase can on its own efficiently degrade small peptides of up to six amino acid residues $[4,5]$. Degradation of longer peptides and proteins required
C Biomed Central

(ㄷ) 2011 Benaroudj et al; licensee BioMed Central Ltd. This is an Open Access article distributed under the terms of the Creative Commons Attribution License (http://creativecommons.org/licenses/by/2.0), which permits unrestricted use, distribution, and reproduction in any medium, provided the original work is properly cited. 
the interaction of ClpP with an ATPase complex, namely ClpA or ClpX in most Gram negative bacteria and ClpX, ClpC, and possibly ClpE and ClpL in Gram positive bacteria [6]. This ATPase complex, a ringshaped hexamer that aligns coaxially with the ClpP peptidase tetradecamer, allows recognition of polypeptide substrates and, through ATP hydrolysis, provides the energy required for protein unfolding and translocation inside the proteolytic chamber where the polypeptide chain is degraded to small peptides.

Mycobacterium tuberculosis (MTB), the etiologic agent of tuberculosis in humans, is one of the deadliest pathogens on earth, killing nearly 2 million people each year [7].

MTB has unique biological properties that enable it to persist in phagosomes for decades in a poorly understood latent form and from where it can be reactivated and cause active tuberculosis [8]. The phagosome is a hostile environment which is acidic, nutrient and oxygen poor, and oxidative and nitrosative due to the production of reactive oxygen and nitrogen species by the host [9]. Consistent with a powerful adaptive capacity, microarray studies have shown that MTB survival in macrophages is accompanied by changes in expression of about 600 genes, many of them involved in starvation, nitrosative and oxidative stress responses [10].

One of the factors potentially involved in the pathogenesis of MTB is the ClpP proteolytic complex, and growing evidences have involved this protease in the virulence of numerous other pathogen bacteria including Salmonella typhimurium [11], Listeria monocytogenes [12], Streptococcus pneumoniae [13], Staphylococcus aureus [14], and Helicobacter pylori [15].

The complete genome sequencing of the best-characterized M. tuberculosis strain (H37Rv) has revealed the presence of two paralog genes encoding putative ClpP peptidase, $c l p P 1$ (Rv2461c) and clpP2 (Rv2460c) (Pasteur Institute TubercuList, http://genolist.pasteur.fr/TubercuList), probably organized as an operon [16].

Presence of multiple clpP genes is common to actinomyces, whereas most other bacteria contain only one clpP gene. $\operatorname{clpP} 2$ has been predicted as essential for growth of MTB by transposon mutagenesis [17]. Both genes have their expression up-regulated during reaeration of MTB cultures [16] and are important for MTB to replicate in macrophages [18]. Despite their potential importance in survival and virulence of MTB, to our knowledge, functional studies of these peptidases have not yet been reported.

In this study, in order to gain insight into the mechanism of protein degradation by ClpPs from MTB, we have produced and purified recombinant ClpP1 and $\mathrm{ClpP} 2$ and tested their peptidase activities. No conventional chymotryptic activity could be detected toward the model peptide Suc-LY-Amc (N-Succinyl-Leu-Tyr-7 amido 4 methylcoumarin) under conditions that normally favor ClpP activity. However, ClpP1 was shown to have a proteolytic activity responsible for its own cleavage after the Arg8 residue and cleavage of $\mathrm{ClpP} 2$ after the Ala12 residue. We have investigated whether the cleavage of model peptides by ClpP1 and ClpP2 would require their proteolytic processing, deletion of the amino termini that could prevent peptide entry, or the presence of the ATPases ClpC and ClpX. We have also tested how the truncations in the amino-termini of the proteins would change their oligomeric assembly and found that they led to a higher order of assembly.

\section{Results}

Recombinant ClpP1 and ClpP2 do not cleave the Suc-LYAmc peptide but can inhibit $E$. coli ClpP activity

The Rv2461c and Rv2460c genes encode proteins of respectively 200 and 214 amino acids annotated as $\mathrm{ClpP} 1$ and ClpP2. Alignment of the primary sequence of those proteins with that of $E$. coli ClpP shows a conservation of the Ser, His, and Asp catalytic triad residues (see additional file 1), indicating that they both have a putative Ser peptidase activity.

In order to study the mycobacterial ClpP peptidase activities, $c l p P 1$ (his) ${ }_{6}, c l p P 2$ (his) ${ }_{6}$ and a $c l p P 1-C l p P 2$ (his) 6 operon were expressed under the control of T7 promoter in E. coli BL21(DE3) cells. When the corresponding proteins were purified by a single $\mathrm{Ni}^{2+}$ affinity chromatography and left for several weeks at $4^{\circ} \mathrm{C}$, all the preparations exhibited a peptidase activity toward the SucLY-Amc fluorogenic peptides (Figure 1A). However, replacing the active site serine residue of ClpP1 (Ser 98) and ClpP2 (Ser 110) by alanine residues did not abolish the observed peptidase activity (data not shown). When ClpP1 and ClpP2 were produced in E. coli cells lacking endogenous ClpP (SG1146a strain), no peptidase activity toward the Suc-LY-AMC peptide was detected (Figure 1A). Also, when an extract was prepared from empty vector-expressing BL21(DE3) cells and subjected to a $\mathrm{Ni}^{2+}$ affinity chromatography, and incubated with the Suc-LY-Amc peptide, no peptidase activity was found (see additional file 2). Therefore, E. coli ClpP rather than MTB ClpP1 and ClpP2 was responsible for the observed peptidase activities and the co-purification of E. coli $\mathrm{ClpP}$ depends on the presence of ClpP1 or ClpP2.

It is noteworthy that not all protein preparations had the same specific peptidase activity toward Suc-LY-Amc nor it could be detected after the same duration of incubation at $4{ }^{\circ} \mathrm{C}$. The proteins purified when the ClpP1$C l p P 2$ (his) 6 operon was overexpressed showed the most rapidly detectable peptidase activity (note that, in the Figure $1 \mathrm{~A}$, only $5 \mu \mathrm{g}$ of $\mathrm{ClpP} 1 / \mathrm{ClpP} 2(\mathrm{His})_{6}$ were used whereas $10 \mu \mathrm{g}$ of the other preparations were used), and 


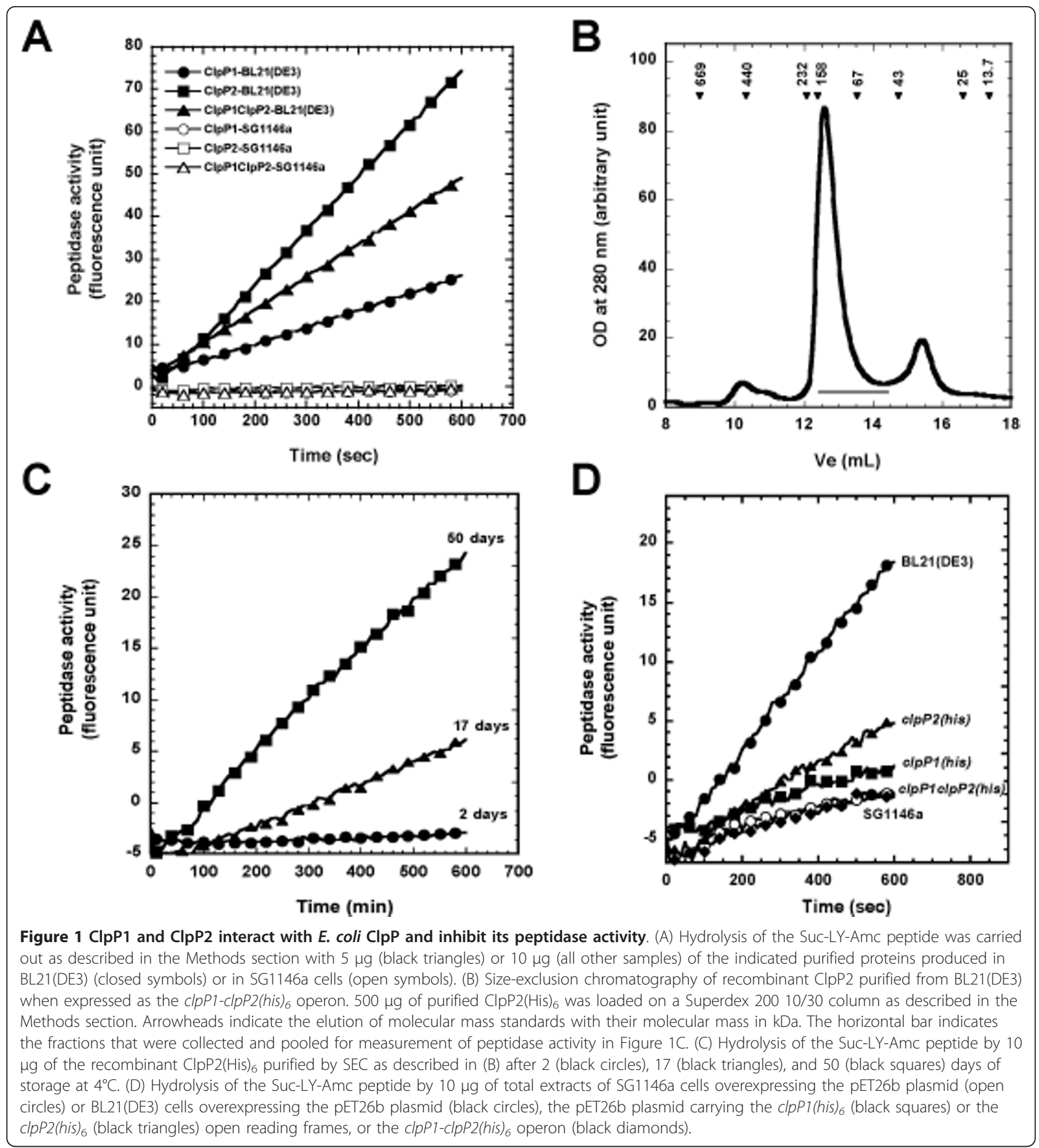

the $\mathrm{ClpP} 1(\mathrm{His})_{6}$ preparation needed to be incubated a longer time at $4^{\circ} \mathrm{C}$ in order to detect $E$. coli $\mathrm{ClpP}$ peptidase activity.

In order to eliminate the contaminating E. coli $\mathrm{ClpP}$ peptidase activity, the proteins purified when the ClpP1$C l p P 2$ (his) 6 operon was overexpressed were subjected to a second chromatographic step. The elution fraction of the $\mathrm{Ni}^{2+}$ column contained mainly $\mathrm{ClpP} 2(\mathrm{His})_{6}$ and a small amount of ClpP1 (data not shown). When subjected to a size exclusion chromatography (SEC), this protein mixture eluted as a main peak with an elution volume of $12.6 \mathrm{~mL}$, which corresponds to a species having an apparent molecular mass of about $104 \mathrm{kDa}$ (Figure 1B). Active E. coli ClpP peptidase assembles in a 
tetradecameric complex of about $300 \mathrm{kDa}$. Therefore, this peak should not contain any active tetradecamer of E. coli ClpP. Fractions corresponding to the mycobacterial ClpP peak were pooled and stored at $4^{\circ} \mathrm{C}$. As seen in Figure $1 \mathrm{C}$, after two days no peptidase activity was detectable. After 17 days of storage at $4^{\circ} \mathrm{C}$, a peptidase activity was measurable and this activity kept increasing after 50 days of storage. Comparable peptidase activity was observed if the protein mixture was not subjected to a SEC but no peptidase activity could be observed if the ClpP1-ClpP2(his) 6 operon was overexpressed in the SG1146a strain and the proteins subjected to a SEC purification step (data not shown). This suggests that the major peak eluting from the SEC column at $12.6 \mathrm{~mL}$ contained, in addition to MTB ClpPs, inactive subunits of $E$. coli ClpP that could reassemble into an active complex upon storage at $4^{\circ} \mathrm{C}$. This would imply that $E$. coli $\mathrm{ClpP}$ subunits could associate with MTB ClpP1 and ClpP2 to form complexes inactive toward the Suc-LYAmc peptide. An interaction between E. coli ClpP and MTB ClpP1 or ClpP2 was demonstrated by co-producing $\mathrm{ClpP} 1(\mathrm{His})_{6}$ or $\mathrm{ClpP} 2(\mathrm{His})_{6}$ with untagged $E$. coli $\mathrm{ClpP}$ and showing that the binding of ClpP to a $\mathrm{Ni}^{2+}$ resin depended on the MTB ClpP1 or ClpP2 (see additional file 3). To verify that ClpP1 or ClpP2, when interacting with $E$. coli $\mathrm{ClpP}$, would inhibit $\mathrm{ClpP}$ peptidase activity, we tested whether expression of ClpP1 and ClpP2 could decrease cleavage of Suc-LY-Amc peptide by E.coli ClpP. As seen in Figure 1D, a peptidase activity toward Suc-LY-Amc peptide was detected in total extract of BL21(DE3) that could be attributed to E. coli ClpP since it dramatically decreased when $\operatorname{clpP}$ was inactivated (SG1146a strain). Expressing $\operatorname{clpP} 1$ (his) ${ }_{6}$, clpP2(his) ${ }_{6}$, or the clpP1-clpP2(his) ${ }_{6}$ operon led to a decrease in $E$. coli ClpP peptidase activity, indicating that MTB ClpPs subunits may interact with E. coli ClpP subunits and inhibit its activity. Such kind of property has never been described for any other recombinant ClpPs produced in E. coli and might reflect a difference in the assembly pathway of protomers into tetradecamers. Our finding that the purified proteins released the active E. coli $\mathrm{ClpP}$ more rapidly when $\operatorname{clpP} 1$ and $\operatorname{clpP} 2$ are expressed from an operon than when MTB ClpPs are produced independently might indicate a stronger, perhaps physiological, interaction between ClpP1 and ClpP2. The copurification of ClpP1 with ClpP2 makes such a hypothesis very likely. Those findings demonstrated the need to produce MTB ClpP1 and ClpP2 in a bacterial strain that does not contain any endogenous $E$. coli ClpP.

Since we could not detect any chymotryptic peptidase activity by MTB ClpP1 and ClpP2 toward the Suc-LYAmc peptide, we also tested cleavage of other peptides. $\mathrm{ClpP} 1$ and $\mathrm{ClpP} 2$, whether produced independently or from the clpP1-clpP2(his) 6 operon, did not cleave the Bz-VGR-Amc (benzoyl-Val-Gly-Arg 7 amido 4 methylcoumarin) or Boc-LRR-Amc (terbutyloxycarbonyl-LeuArg-Arg 7 amido 4 methylcoumarin) peptides (data not shown), indicating that they do not exhibit trypsin-like activity toward those peptides in the conditions used in our assay.

\section{Recombinant MTB ClpP1 and ClpP2 do not assemble into tetradecamers in solution}

One of the hypotheses to explain the absence of any peptidase activity observed in our assay is the absence of a tetradecameric assembly. Therefore, the presence of a correct tetradecameric assembly was examined on purified ClpP1 and ClpP2. When subjected to SEC, purified $\mathrm{ClpP} 1$ eluted as a species having an apparent molecular mass of $35 \mathrm{kDa}$, compatible with monomers and dimers of ClpP1 (Figure 2, upper panel, solid trace). Purified ClpP2 eluted as a molecular complex having an apparent molecular mass of about $91 \mathrm{kDa}$ (Figure 2, middle panel, solid trace). These results indicated that neither ClpP1 nor ClpP2 assembled in tetradecamers in these conditions.

The possibility that both $\mathrm{ClpP} 1$ and ClpP2 were needed to promote tetradecamer assembly was tested. As seen in Figure 2 (upper panel, dashed line), coproducing ClpP2 with ClpP1 did not dramatically change the elution volume of purified ClpP1 and similarly coproducing ClpP1 with ClpP2 did not influence the elution volume of ClpP2 (middle panel, dashed line). Furthermore, mixing purified ClpP1 and ClpP2 did not change the elution volumes of individual ClpP1 or ClpP2 (Figure 2, lower panel). Therefore, tetradecameric assembly was not mutually induced by the presence of either ClpP1 or ClpP2.

It is noteworthy that the presence of a His tag at the C-termini of ClpP1 and ClpP2 could not explain the absence of tetradecamer formation since producing and purifying untagged $\mathrm{ClpP} 1$ and $\mathrm{ClpP} 2$ led to the same oligomeric assembly as His tagged peptidases (data not shown).

\section{Peptidase activity and assembly of processed ClpP1 and ClpP2}

We then explored different procedures to stimulate the peptidase activity and to promote correct tetradecameric assembly of ClpP1 and ClpP2.

In E. coli, ClpP matures as an active peptidase by the autoproteolytic removal of the first 14 amino acid residues $[19,20]$. In the X-ray structure of MTB ClpP1, the first 14 residues are not visible and it was suggested that the $\mathrm{N}$-terminus of the protein was disordered and might prevent the entry of small peptides into the central proteolytic cavity, explaining the lack of peptidase activity 
column. Arrowheads indicate the elution of molecular mass standards with their molecular mass in kDa and the deduced apparent molecular masses are shown under the corresponding protein names.

[21]. We thus tested whether processed ClpP1 and ClpP2 would correctly assemble in tetradecamers and exhibit chymotryptic activity.

Processed E. coli ClpP has been shown to start with Ala15 [19]. Based on alignment with E.coli ClpP, ClpP1 is not expected to be processed (Figure 3). If aligned with the whole sequence of Streptomyces ClpP1 (a closer ortholog), it could be processed at Met7. However, it was shown that Streptomyces ClpP1 had another putative initiation codon according to the codon usage [22] and if aligned with the streptomyces ClpP1 sequence starting with this second putative initiation codon MTB ClpP1 would be processed at the Ser9 residue (Figure 3).

Based on alignement with E.coli ClpP, ClpP2 is predicted to be processed at Tyr14. In Streptomyces, mature ClpP2 was shown to start at Val37 [22], which would predict that mature MTB ClpP2 starts with the Ile15 residue (Figure 3).

Several truncated variants of ClpP1 starting with Met7 (M7ClpP1) or Ser9 (S9ClpP1), and of ClpP2 starting with Arg13 (ClpP2R13), Tyr14 (ClpP2Y14), Ile15 (ClpP2I15), or Leu16 (ClpP2L16) residues were produced and purified in SG1146a cells (see additional file 4 ), and analyzed for peptide cleavage and oligomeric assembly.

Removing the putative prosequences of ClpP1 did not change its elution during SEC (data not shown). Likewise, most of the processed ClpP2 variants eluted with an elution volume comparable to that of wild-type ClpP2 (data not shown). However, the ClpP2R13 variant surprisingly eluted as three main species. In order to determine precisely the nature of these different oligomeric species, full-length ClpP2 and the ClpP2R13 variant were subjected to size-exclusion chromatography coupled online to a triple detector array (SEC-TDA) and further analyzed by static light scattering measurements. In SEC-TDA experiment, the analysis of full-length ClpP2 peak indicated the presence of a range of assemblies with a weight average molecular mass of $140 \mathrm{kDa}$ compatible with hexamers or heptamers of ClpP2 subunits (Figure 4, upper panel). Analysis of the ClpP2R13 variant by SEC-TDA indicated species with molecular masses of $482.5 \mathrm{kDa}, 148.0 \mathrm{kDa}$ and $46.3 \mathrm{kDa}$ corresponding to 21 -mer, heptamer, and dimer species respectively (Figure 4 , lower panel). The 21 -mer oligomeric species was also observed during the elution of the ClpP2Y14, but in a lower amount (data not shown). This high molecular weight complex is reminiscent of
Figure 2 Oligomeric assembly of recombinant ClpP1 and ClpP2. $75 \mu \mathrm{g}$ of recombinant ClpP1 (upper panel, solid line) or ClpP2 (middle panel, solid line) produced independently or in the presence of ClpP2 (upper panel, dashed line) and ClpP1 (middle panel, dashed line) respectively were loaded on a superdex 200 10/ 30 column as described in the Methods section. In the lower panel, $75 \mu \mathrm{g}$ of ClpP1 were mixed with $75 \mu \mathrm{g}$ of ClpP2 and incubated $2 \mathrm{~h}$ at room temperature before being loaded on the superdex 200 


\begin{tabular}{|c|c|}
\hline EcClpP & MSYSGERDNFAPHMALVPMVIEQTSRGERSFDIYSRLLKERVIFLTGQVEDHMANLIVAQ \\
\hline TBClpP1 & $---M S Q V T D M R S N S Q G L S L T D S V Y E R L L S E R I$ IFLGSEVNDE IANRLCAQ \\
\hline TBClpP2 & $\begin{array}{c}\text {-MNSQNSQIQPQARY ILPSF IEHSSFGVKESNPYNKLFEERI IFLGVQVDDASANDIMAQ } \\
*\end{array}$ \\
\hline
\end{tabular}

SeclpP 1

MRRPGAVVRRAGGYVTNLMPSAAGEPSIGGGLGDQVYNRLLGERI IFLGQPVDDDIANKI 60

TBClpP 1 MSQVTDMRSNSQGLSLTDSVYERLLSERIIFLGSEVNDEIANRL 44

SeClpP 1

MTNLMPSAAGEPSIGGGLGDQVYNRLLGERI IFLGQPVDDDIANKITAQLLLLAS-DPDK 59

TBClpP1 MSQVTDMRSN--SQGLSLTDSVYERLLSERI IFLGSEVNDE IANRLCAQILLLAAEDASK 58

SeClpP2

MNDFPGSGLYDRVNAAQDMRAASQGRYTGPQAESRYVIPRFVERTSQGVREYDPYAKLF 59

TBClpP2

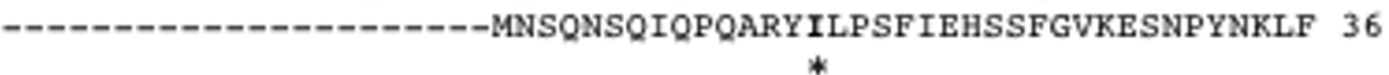

Figure 3 Putative $\mathrm{N}$-terminal processing sites for MTB ClpP1 and ClpP2. The N-terminal sequences of MTB ClpP1 (H37Rv strain, gi: 41353667) and ClpP2 (H37Rv strain, gi: 2791500) were aligned with that of E. coli ClpP (gi: 89107307) and with those of S. coelicolor ClpP1 (gi: 10280519) and ClpP2 (10280518) respectively using ClustalW program http://www.ebi.ac.uk/Tools/msa/clustalw2. The propeptides of E. coli ClpP and Streptomyces ClpP1 and ClpP2 are written in italics and the first residues of mature ClpPs are indicated in bold. The putative first residues of MTB ClpP1 and ClpP2 are written in bold and indicated with an asterisk.

that observed with E. coli ClpP in the absence of salt [19] and could correspond to three stacked heptameric rings. Therefore, removing the putative prosequence of ClpP2 did promote the formation of higher order oligomers but not of the expected tetradecamer. None of those ClpP1 or ClpP2 variants exhibited any activity toward Suc-LY-Amc or Boc-LRR-Amc peptides (data not shown). Thus, removing putative prosequences was not sufficient for allowing ClpP1 and ClpP2 to assemble into tetradecamers with activity toward model peptides.

\section{Deleting the entry gate does not activate ClpP1 and ClpP2}

Since the N-terminal extremity of ClpP2 influenced its oligomerisation state, we tested whether further deletions in their N-termini would favor assembly of ClpP1 and ClpP2 in tetradecamer.

Recent studies have demonstrated a gating mechanism for $E$. coli ClpP comparable to that of proteasomes, a self-compartmentalized protease with a similar barrelshaped architecture. In general, the first 20 residues of mature ClpP (after its processing) adopt a flexible conformation that can explain why they are not always visible in electron density map. In the mature processed ClpP, the
$\mathrm{N}$-terminal extremity can adopt different secondary structures and therefore modulate the size of the entry pore [23]. In the "up" conformation, a portion of the Nterminus extends outwards the access pore whereas in the "down" conformation it is located within the axial pore. For E. coli ClpP, removing the residues involved in the gating mechanism allowed degradation of unfolded substrates in the absence of the ATPase complex [24]. For the eukaryotic $20 \mathrm{~S}$ proteasomes, the entry gate precludes entry of even small size peptides [25].

In MTB ClpP1, the residues Ser15-Glu27 fold as a helix that is longer than the corresponding helix in ClpP orthologues and that protruded into the axial pore of the tetradecamer, making a gating mechanism likely [21]. To see whether removing the putative gate in MTB ClpP1 and ClpP2 would have an effect on peptide cleavage, $\mathrm{N}$-terminal gate deletion variants were constructed based on the alignment with E. coli ClpP (Figure $5 \mathrm{~A}$ ). In $E$. coli, deletion of the first 10, 14, and 17 first amino acids (based on the numbering of mature $\mathrm{ClpP}$ ) activated degradation of unfolded substrates to a different extent, with the $\Delta 14 \mathrm{ClpP}$ variant being the most active. Corresponding deletion variants were produced and their peptidase activity and tetradecameric 

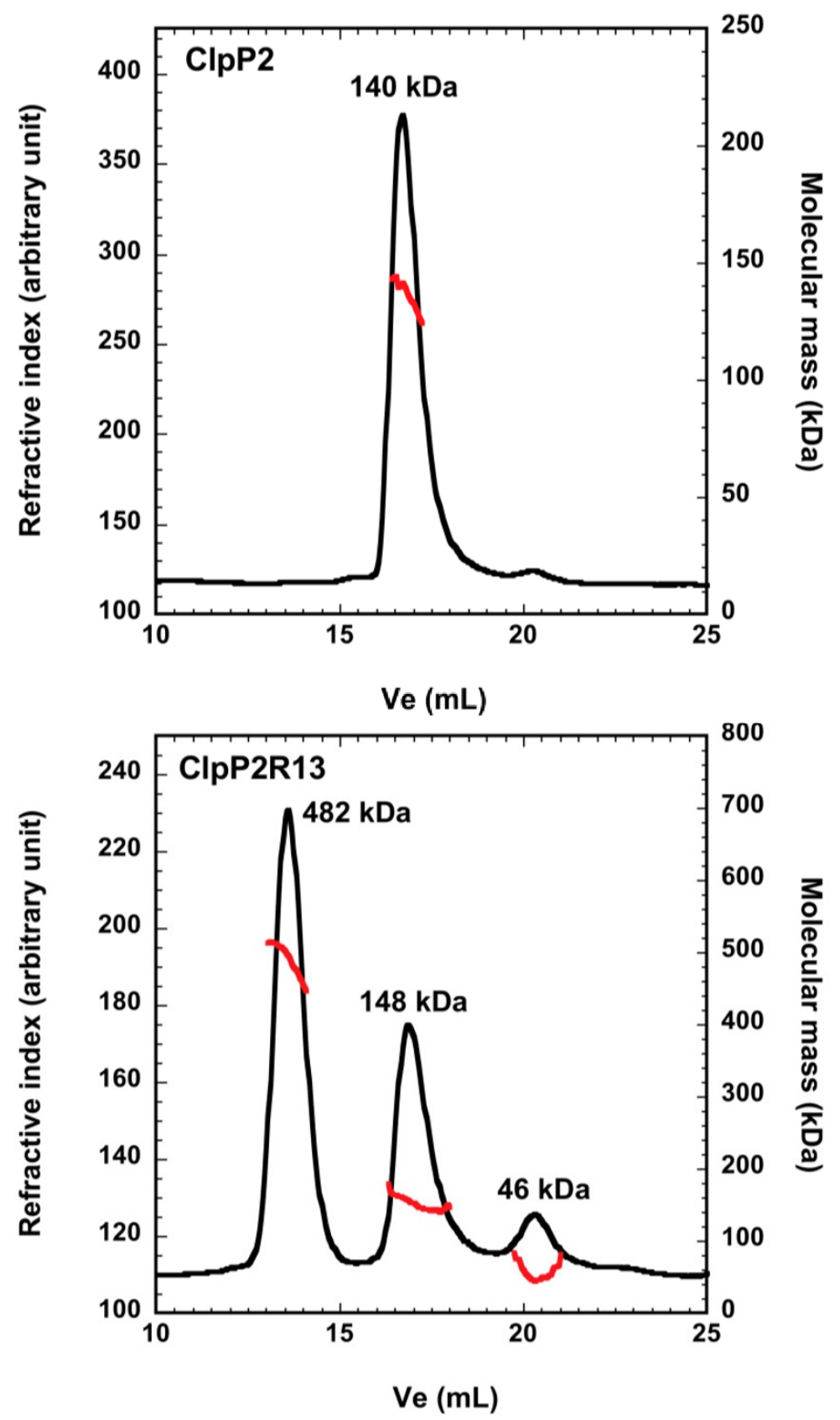

Figure 4 Effect of removing putative propeptide on the assembly of ClpP2. $200 \mu \mathrm{g}$ of purified ClpP2 (upper panel) or of the ClpP2R13 variant (lower panel) were subjected to a size exclusion chromatography as followed by a triple detector array as described in the Methods section. The refractive index (black line) and the molecular mass (red line) were plotted as a function of the elution volume. Average molecular weights obtained by static light scattering are indicated at the top of each peak.

assembly were tested. None of them exhibited any significant peptidase activity toward the Suc-LY-Amc peptide (data not shown), suggesting that the absence of chymotryptic activity toward this peptide in WT ClpP1 and ClpP2 was not due to any obstruction of the pore entrance by their $\mathrm{N}$-termini.
When we examined their assembly by SEC-TDA, we found that when the first 15 residues of ClpP1 were absent, an additional peak was detected. Indeed, fulllength ClpP1 exhibited the presence of a several assemblies with molecular masses ranging from 30 to 70.5 $\mathrm{kDa}$ compatible with complexes varying from monomers 


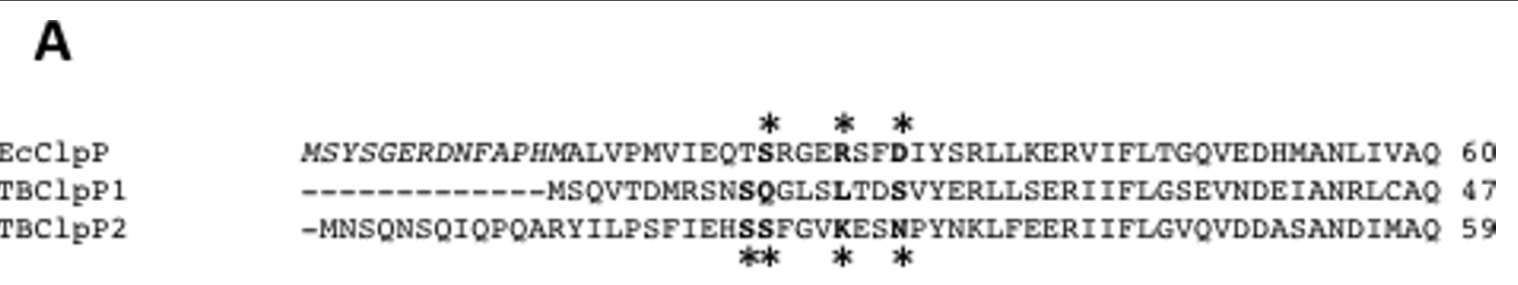

B
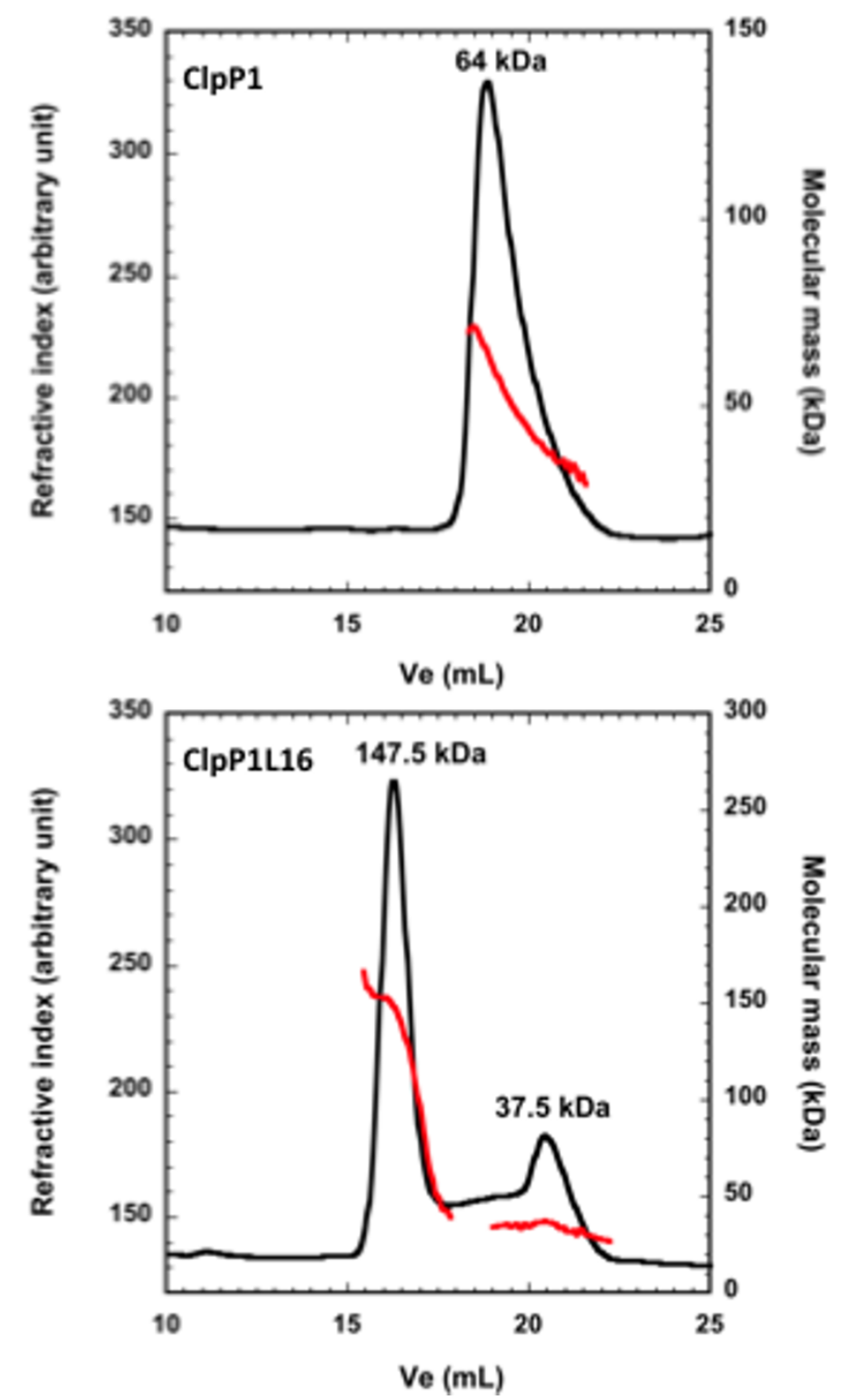

Figure 5 Effect of removing the putative gate on the assembly of mature ClpP1. (A) The N-terminal sequences of MTB ClpP1 (H37Rv strain, gi: 41353667) and ClpP2 (H37Rv strain, gi: 2791500) were aligned with that of E. coli ClpP (gi: 89107307). The propeptide of E. coli ClpP is written in italics. The first residues of deletion variants of $E$. coli ClpP are indicated in bold and by an asterisk above the sequence. The corresponding residues in MTB ClpP1 and ClpP2 sequences are written in bold and indicated with an asterisk below their sequences. (B) $200 \mu \mathrm{g}$ of purified ClpP1 (upper panel) or of the ClpP1L16 variant (lower panel) were subjected to a size exclusion chromatography as followed by a triple detector array as described above. 
to trimers of $\mathrm{ClpP} 1$ subunits (Figure 5B, upper panel). The ClpP1L16 variant eluted as a sharper peak corresponding to a species having a molecular mass complex of about $150 \mathrm{kDa}$, compatible with $\mathrm{ClpP} 1$ heptamers (Figure 5B, lower panel). Thus, removing the $\mathrm{N}$-terminal extremity of ClpP1 likely favors assembly of the ClpP1 heptamericring.

Deleting the amino terminal residues of $\mathrm{ClpP} 2$ slightly decreased its elution volume during SEC but did not promote higher order oligomeric assembly above heptameric form (data not shown). These finding indicated that further removing the $\mathrm{N}$-terminal extremities of ClpP1 and ClpP2 could stabilize the heptamericring.

\section{Coproduction of the peptidases with the ATPases ClpC1 and ClpX}

Most of ClpPs, when tested, are able to degrade small peptides in the absence of the ATPase complex. One exception is the human mitochondrial ClpP that required the ATPase ClpX to assemble into stable tetradecamer with a high peptidase activity [26]. In the MTB genome, three genes have been annotated as Clp/hsp100 proteolysis associated ATPase, $\operatorname{clpC1}$ (Rv3596c), $\operatorname{clp} C 2$ (Rv2667), and $\operatorname{clpX}$ (Rv2457c) (Pasteur Institute TubercuList, http://genolist.pasteur.fr/TubercuList).

Primary sequences of $\mathrm{ClpC} 1$ and $\mathrm{ClpX}$ contain the typical Clp ATPase features, $i$. e. one or two AAA (A-TPase Associated with various cellular Activities) domains. However, $\mathrm{ClpC} 2$ protein does not exhibit any AAA or other ATPase motif and the purified $\mathrm{ClpC} 2$ did not have any ATPase activity (unpublished data). The presence of two ClpN domains in the $\mathrm{ClpC} 2$ sequence might have been at the origin of the mistaken annotation of this open reading frame as a Clp ATPase. Therefore, only $\mathrm{ClpC} 1$ and $\mathrm{ClpX}$ seem to be putative ATPase partners for $\mathrm{ClpP} 1$ and $\mathrm{ClpP} 2$ peptidases. To investigate whether MTB ClpP1 and ClpP2 require their ATPase partners to hydrolyze peptides, ClpP1 and ClpP2 were produced with $\mathrm{ClpC}$ or $\mathrm{ClpX}$ (Figure 6A) and peptide cleavage was tested in total extracts. The presence of $\mathrm{ClpX}$ or ClpC did not result in Suc-LY-Amc hydrolysis (Figure 6B). Therefore, the absence of Suc-LY-Amc cleavage by $\mathrm{ClpP} 1$ and $\mathrm{ClpP} 2$ was not due to a requirement for the ATPase complex.

\section{ClpP1 is responsible for ClpP1 and ClpP2 processing}

When produced in E. coli, ClpP1 migrated as two bands on SDS-PAGE (Figure 7A, lane 1). Microsequencing revealed that the upper band contained a protein starting with SQVTDM, corresponding to a full-length ClpP1 that had its initiating methionine residue removed. The smaller protein in ClpP1 preparation (labeled * in Figure 7A) started with SNSQG, corresponding to ClpP1 starting at the Ser9 residue. When the Ser catalytic residue of ClpP1 was replaced by an Ala (Ser98Ala variant), this form of ClpP1 was not visible (Figure 7A, lane 2). Therefore, proteolytic activity of ClpP1 was responsible for its own cleavage.

ClpP2 migrated mainly as a single band on SDS-PAGE (Figure 7A, lane 3). Microsequencing showed that it corresponded to the full-length ClpP2 (starting with MNSQNS residues). When ClpP2 was coproduced with ClpP1, two bands were obtained (Figure 7A, lane 4). The protein of the upper band started with MNSQNS residues and corresponded to full-length $\mathrm{ClpP} 2$. The protein in the lower band (labeled $* *$ in Figure 7A and 7B) started with RYILP and corresponded to ClpP2 starting at the Arg13 residue. Therefore, coproducing $\mathrm{ClpP} 1$ and $\mathrm{ClpP} 2$ led to processing of ClpP2. In order to test which peptidase was responsible for $\mathrm{ClpP} 2$ processing, we inactivated $\mathrm{ClpP} 1$ and $\mathrm{ClpP} 2$ by replacing their active site serine by an alanine residue (ClpP1Ser98Ala and ClpP2Ser110Ala) in the clpP1-clpP2(his) 6 operon. Inactivating $\mathrm{ClpP} 2$ only did not prevent its processing (Figure 7B, lane 3) whereas ClpP2 processing was not observed when ClpP1 was inactivated (Figure 7B, lane 2). Altogether, these results demonstrated that recombinant $\mathrm{ClpP} 1$ possessed a proteolytic activity responsible for its own cleavage after the Arg8 residue and for $\mathrm{ClpP} 2$ cleavage after the Ala12 residue. It is noteworthy that the truncated ClpP1 form produced by the proteolytic activity of ClpP1 corresponded to the putative processed ClpP1 as predicted by sequence alignment. In $\mathrm{ClpP} 2$, the processing cleavage site catalyzed by ClpP1 was in the vicinity of that predicted by sequence alignment. In conclusion, even though no Suc-LY-amc cleavage was observed by ClpP1, this peptidase exhibited the ability to hydrolyze a peptide bond.

\section{Discussion}

Most of our knowledge on the mechanism of protein degradation by ClpP protease is based on studies of $E$. coli ClpP. This peptidase is able alone to hydrolyze the model dipeptide Suc-LY-Amc in vitro [5]. In this study, we have shown that, in conditions that normally allow Suc-LY-Amc cleavage by E. coli ClpP, MTB ClpP1 and $\mathrm{ClpP} 2$ do not hydrolyze this model peptide. However, a proteolytic activity was uncovered for $\mathrm{ClpP} 1$, ruling out the possibility that, at least for $\mathrm{ClpP} 1$, those peptidases were produced in E. coli as totally inactive.

An absence of peptidase activity was not due to the presence of a His tag at the C-termini of ClpP1 or $\mathrm{ClpP} 2$ since purifying those peptidases without any tag did not allow hydrolysis of Suc-LY-Amc or of other model peptides, nor it promoted correct tetradecameric assembly.

A failure to detect model peptide cleavage was not due to an obstruction of the entry pore by the $\mathrm{N}$ - 


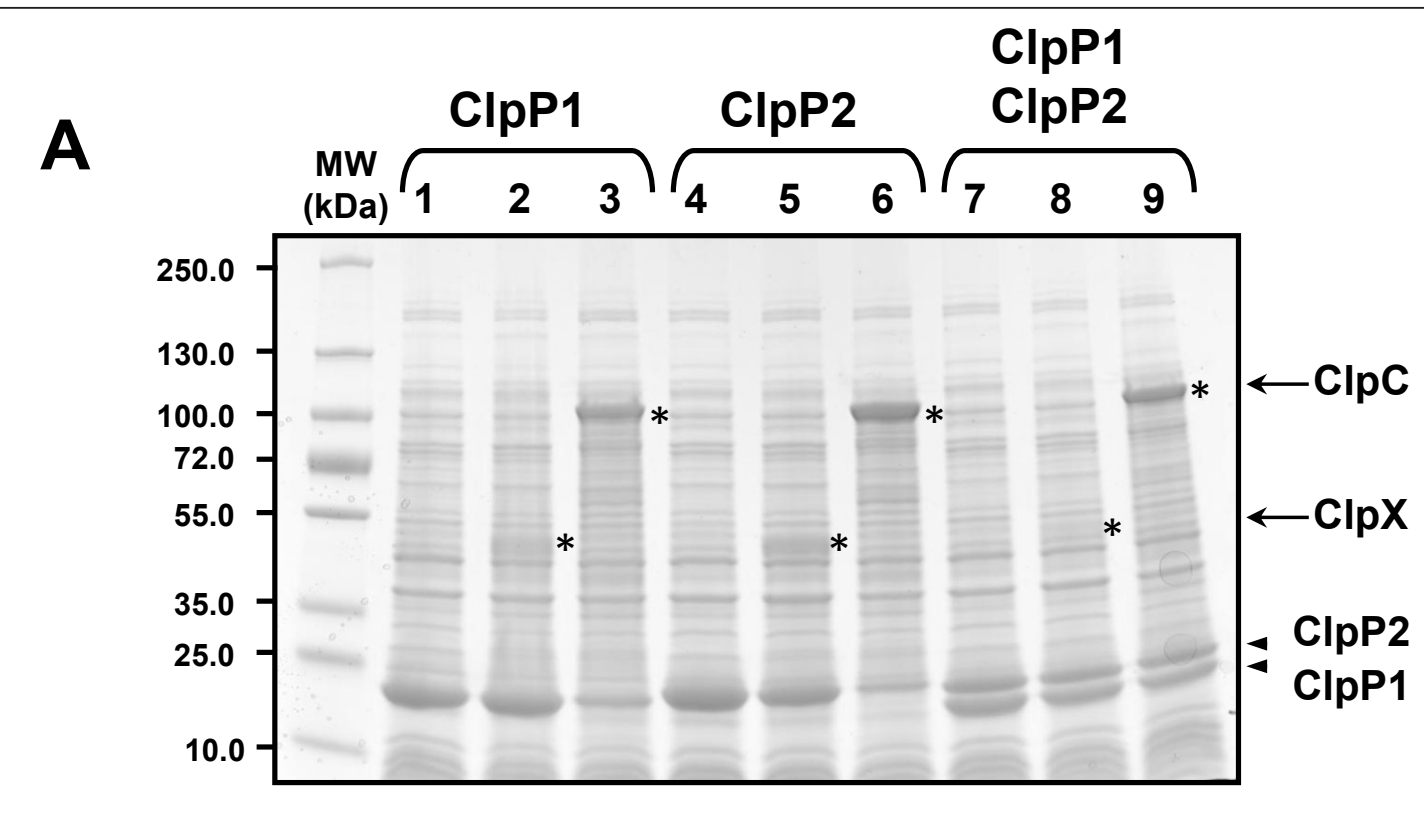

B
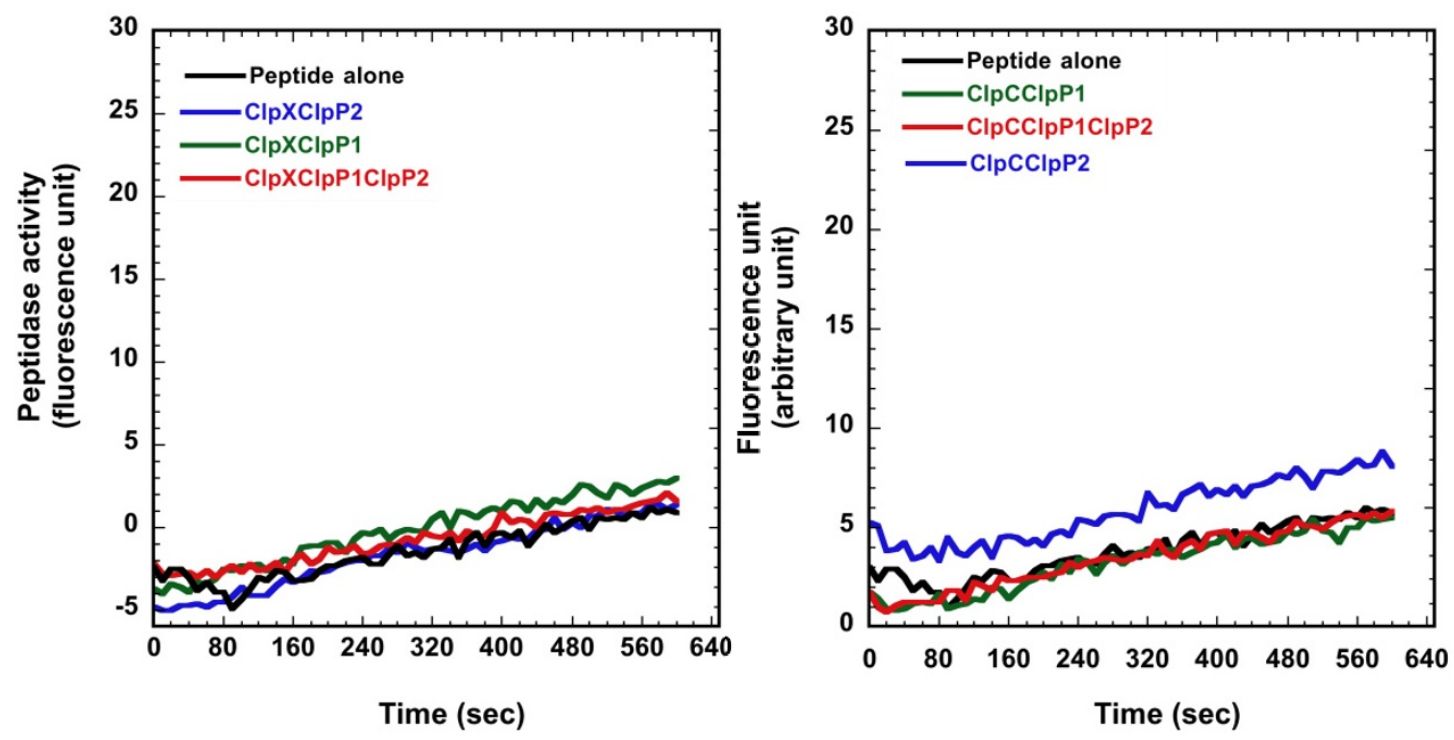

Figure 6 Coproducing ClpP1 and ClpP2 with ClpX and ClpC ATPases did not result in Suc-LY-Amc cleavage. (A) $10 \mu \mathrm{g}$ of total extract of cells producing ClpP1 (lane 1), ClpP1 and ClpX (lane 2), ClpP1 and ClpC (lane 3), ClpP2 (lane 4), ClpP2 and ClpX (lane5), ClpP2 and ClpC (lane 6), ClpP1 and ClpP2 without (lane 7) or with ClpX (lane 8) or ClpC (lane 9) were loaded on a 4-15\% gradient SDS-PAGE stained with coomassie blue. The electrophoretic mobilities of ClpX and ClpC are indicated by asterisks. The molecular masses of the markers were indicated on the left in $\mathrm{kDa}$. (B) Hydrolysis of the Suc-LY-Amc peptide in the absence (black trace) or in the presence of $10 \mathrm{\mu g}$ of total extracts of SG1146a cells overexpressing $c \mid p P 1$ (his) $)_{6}$ and $c \mid p X$ (left panel, green trace), $c \mid p P 2$ (his) $)_{6}$ and $c l p X$ (left panel, blue trace), the clpP1-clpP2(his) 6 operon together with

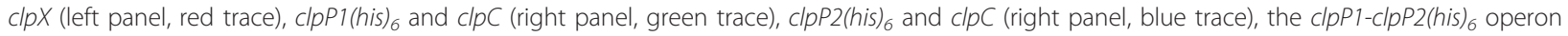
together with $c \mid p C$ (right panel, red trace).

terminal extremities of $\mathrm{ClpP} 1$ and $\mathrm{ClpP} 2$, predicted to be highly flexible, since removing these extremities did not result in peptide cleavage. Furthermore, coproducing the ClpX and ClpC ATPase complexes with ClpP1 and ClpP2 did not allow Suc-LY-Amc cleavage, showing that an absence of peptide model hydrolysis by mycobacterial ClpPs was not due to an obligatory requirement for the ATPases.

One plausible explanation for an absence of Suc-LYAmc cleavage would be that mycobacterial ClpPs 


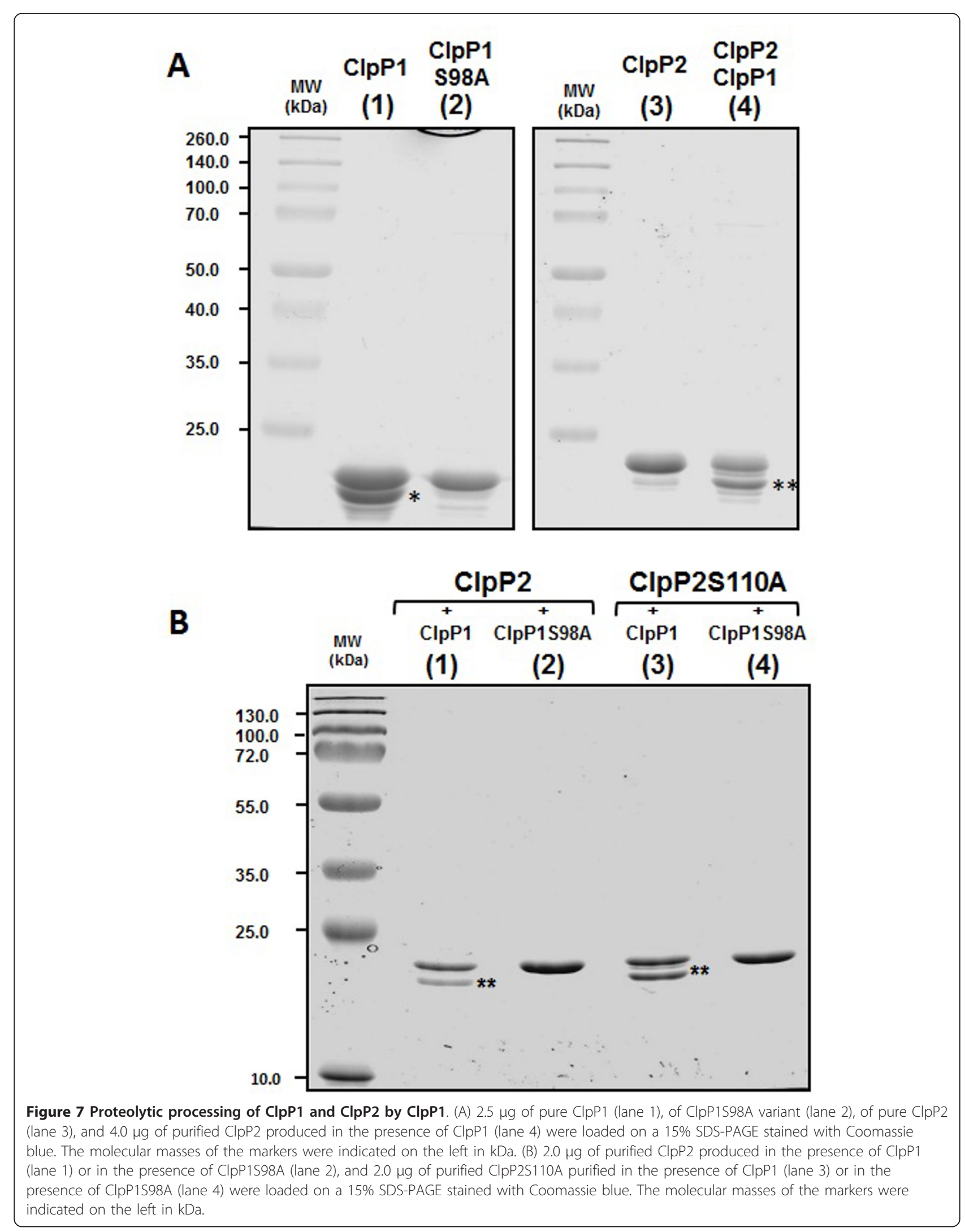


require different physico-chemical conditions to hydrolyze this peptide than those needed by $E$. coli ClpP. In fact, based on crystal structure determination, the tetradecamer of ClpPs from different organisms could be grouped into two structural states: an extended state fully active toward the Suc-LY-Amc model peptide (seen with E. coli, Homo sapiens, H. pylori ClpPs) and a more compact state that likely corresponds to an inactive state (seen with M. tuberculosis, S. pneumoniae, Plasmodium falciparum) [27]. Recombinant ClpP1 and ClpP2 might be isolated in the compact state and require specific physico-chemical conditions to switch to the extended fully active conformation toward model peptides.

Also, the specificities of peptide bond hydrolysis could be different than those of E. coli ClpP. Indeed, the nature of the amino acid in the $\mathrm{P} 1$ position relative to the scissile peptide is important in controlling the hydrolysis rate. For instance, E. coli $\mathrm{ClpP}$ has been shown to exhibit the greatest degradation rate when a large aromatic amino acid residue is in the P1 position (as in the SucLY-Amc peptide) [28].

However, this feature is not shared by all ClpPs since P. falciparum ClpP exhibited a preference for an Arg residue in P1 position [29]. Likewise, MTB ClpP1 and $\mathrm{ClpP} 2$ could require another amino acid residue at the P1 position. Consistent with such a hypothesis is our finding that ClpP1 can cleave a peptide bond after Ala and Arg residues. In the light of these findings, short peptides of those specificities (H-A-Amc, Suc-AAAAmc, H-GR-Amc, Boc-LRR-Amc, Bz-VGR-Amc) were tested but none of them were cleaved by ClpP1 or ClpP2. Determination of the peptide specificities of MTB ClpPs must await further studies.

In this study, we have also shown that recombinant MTB ClpP1 and ClpP2 could not assemble as tetradecamers in solution. Determining the X-ray structure of ClpP1 has shown that ClpP1 could assemble in a tetradecamer under the crystal conditions but the interactions between the two heptamers that stabilize the tetradecamer were weaker than those in other ClpPs; and $\mathrm{ClpP} 1$ was mainly isolated as a heptamer in solution [21]. Our findings suggest that a weak interaction between heptamers could also apply for ClpP2 assembly. In our study, we showed that deleting the amino-terminal extremity of ClpP1 and ClpP2 favored a higher order assembly. In most of the deposited X-ray structures, the amino-terminal extremity of mature $\mathrm{ClpP}$ is unmodelled because non interpretable in the electron density. This suggested a high flexibility in this portion of the protein. Indeed, Bewley et al. [23] demonstrated that the first 20 residues in the mature E. coli $\mathrm{ClpP}$ could adopt two different conformations. In the X-ray structures of the unprocessed full length MTB ClpP1, the first 15 residues were not visible and the Ser15Glu27 portion formed a $\alpha$-helix longer than in other orthologous ClpPs that partially occupies the axial pore [21]. The flexibility of this might hinder a correct assembly of mycobacterial ClpPs and a deviation in its conformation form other orthologous ClpPs might explain a difference in the stabilization of the tetradecamer. Whether an interaction of mycobacterial ClpPs with the ATPase ClpX and ClpC could stabilize a tetradecameric assembly remains to be tested.

Despite that recombinant ClpP1 did not assemble in a tetradecamer, it exhibited a proteolytic activity that was reminiscent of the autocatalytic processing of $\mathrm{ClpP}$ proteases. Autocatalytic processing in ClpP might not require the tetradecameric functional assembly required for the processive proteolytic activity of ClpP. Indeed, the minimal functional structure for the hydrolytic activity of $\mathrm{ClpP}$ was found to be a single heptameric ring [30].

\section{Conclusion}

In this study, we have uncovered an unconventional mechanism of oligomeric assembly and proteolytic activity for MTB ClpP1 and ClpP2 that are distinct from those of other ClpPs, especially the well-characterized $E$. coli ClpP. A better knowledge of such ATP-dependent proteases which are potentially important for the survival and virulence of MTB could offer new hope in the need for developing new drug to treat tuberculosis.

\section{Methods}

Bacterial strain and primers

E. coli BL21(DE3) [31] (Novagen) and BL21(DE3) ClpP:: cam (SG1146a) (Susan Gottesman) strains were used to express recombinant proteins. All primers used are listed in the additional file 5 .

\section{Cloning of clpP2 (Rv2460c) and clpP1 (Rv2461c) ORFs}

clpP1 ORF (Rv2461c) was amplified by PCR and cloned into the pET26b vector (Novagen) using the ClpP15Nde and ClpP1-3Xho primers (see additional file 5) at the NdeI and XhoI sites, giving rise to the pNB71 plasmid containing the $c l p P 1$ ORF with a C-terminal (His) 6 tag. clpP2 ORF (Rv2460c) was amplified by PCR using the ClpP2-5Nde and ClpP2-3HindChis primers and cloned at the NdeI and HindIII sites into the pet26b vector, giving rise to the pNB74 plasmid in which the clpP2 ORF was C-terminal (His) 6 tagged.

The plasmid expressing the clpP1-clpP2(his) 6 operon was constructed by first amplifying the $\operatorname{clpP1}$ ORF with the ClpP1-5Nde and ClpP1-3Hind primers and cloned at the NdeI and HindIII sites into the pET26b vector. The resulting plasmid was named pNB70. The $c l p P 2$ (his) 6 ORF was amplified by PCR from the pNB74 plasmid using the rbsClpP2Hind and ClpP2-3Not primers 
and introduced at the HindIII and NotI sites into the pNB70 plasmid. The resulting plasmid was named pNB82. clpP1 C-terminal (His) 6 tagged ORF starting at the Met7, Ser9, Ser11, Gln12, Leu16, and Ser 19 residues were obtained by PCR amplification using the ClpP1M7, ClpP1S9, ClpP1S11, ClpP1Q12, ClpP1L16 and ClpP1S19 primers respectively as well as the ClpP1$3 \mathrm{Xho}$ primer and introduced at the NdeI and Xhol sites into the pET26b vectors. clpP2 C-terminal (His) ${ }_{6}$ tagged ORF starting at the Arg13, Tyr14, Ile15, Leu16, Ser 23, Ser24, Lys28, and Asn 31 residues were obtained by PCR amplification using the ClpP2R13, ClpP2Y14, ClpP2I15, ClpP2L16, ClpP2S23, ClpP2S24, ClpP2K28, and ClpP2N31 primers respectively as well as the $\mathrm{ClpP} 2-\mathrm{CH}$ is primer and introduced at the NdeI and HindIII sites into the pET26b vector. DNA sequence of all open reading frames was checked by DNA sequencing (Beckman Coulter Genomics).

\section{Protein production and purification}

Cells were cultivated at $30^{\circ} \mathrm{C}$ in Luria-Bertani (LB) medium supplemented when necessary with $30 \mu \mathrm{g} / \mathrm{mL}$ of kanamycin and $25 \mu \mathrm{g} / \mathrm{mL}$ of chloramphenicol. BL21 (DE3) or SG1146a cells were transformed with an expression vector carrying the $c l p P 1$ and/or $c l p P 2$ ORFs and cultivated until an $\mathrm{OD}_{600}$ of 0.6. Protein expression was then induced with $1 \mathrm{mM}$ isopropyl- $\beta$-D-thiogalactopyranoside (IPTG). After centrifugation, cell pellets were resuspended in the buffer A $\left(50 \mathrm{mM} \mathrm{NaH}_{2} \mathrm{PO} 4 \mathrm{pH} 8.0\right.$, $300 \mathrm{mM} \mathrm{NaCl}, 10 \mathrm{mM}$ Imidazole, $10 \%$ glycerol) and lysed by sonication. The soluble fraction obtained after a 60 minutes centrifugation at $40,000 \mathrm{~g}$ at $4^{\circ} \mathrm{C}$ was loaded on nickel-nitrilotriacetic (Ni-NTA) resin (Qiagen) and the resin was washed with buffer A. His-tagged proteins were eluted with buffer B $\left(50 \mathrm{mM} \mathrm{NaH}_{2} \mathrm{PO} 4 \mathrm{pH}\right.$ 8.0, $300 \mathrm{mM} \mathrm{NaCl}, 250 \mathrm{mM}$ Imidazole, 10\% glycerol). After elution, His-tagged proteins were dialyzed against 50 $\mathrm{mM}$ Tris- $\mathrm{HCl} \mathrm{pH}$ 7.5, $200 \mathrm{mM} \mathrm{KCl}, 2 \mathrm{mM}$ DTT, 0.1 mM EDTA, 10\% glycerol and concentrated. Protein concentrations were determined with Coomassie Plus Protein Assay Reagent (Thermo Scientific).

\section{Inactivation of ClpP1 and ClpP2}

The active site Ser residues in ClpP1 (Ser98) and in ClpP2 (Ser110) were replaced by an Ala residue using the Quick Change Multi Site-Directed Mutagenesis kit (Stratagene) according to the manufacturer's recommendation with the ClpP1S98A and ClpP2S110A primers respectively. $\mathrm{ClpP} 1$ and $\mathrm{ClpP} 2$ variants were produced and purified in SG1146a cells as described above.

\section{Peptide hydrolysis}

$1 \mathrm{mM}$ of fluorogenic Suc-LY-Amc peptide diluted in $100 \%$ DMSO was incubated with purified ClpP1 or
ClpP2 or with total extracts as indicated at $37^{\circ} \mathrm{C}$ in 50 $\mathrm{mM}$ Tris $\mathrm{pH}$ 7.5, $100 \mathrm{mM} \mathrm{KCl}$ and $1 \mathrm{mM}$ DTT. Hydrolysis of the peptide was followed by measuring the release of amc (7-amino-4-methylcoumarin) in a spectrofluorometer $\left(\lambda_{\text {ex }} 380 \mathrm{~nm} ; \lambda_{\text {em }} 460 \mathrm{~nm}\right)$.

\section{Size exclusion chromatography}

SEC was carried out at room temperature on a Superdex 200 10/30 column (GE Healthcare) equilibrated with 25 $\mathrm{mM}$ Tris- $\mathrm{HCl}, \mathrm{pH} 7.5,150 \mathrm{mM} \mathrm{KCl}$, and $1 \mathrm{mM}$ DTT. Elution was performed using the same buffer at a flow rate of $0.5 \mathrm{~mL} / \mathrm{min}$, and absorbance was measured at 280 $\mathrm{nm}$. The column was calibrated with Ferritin $(440 \mathrm{kDa})$, Catalase $(232 \mathrm{kDa})$, Aldolase $(158 \mathrm{kDa})$, Bovin Serum Albumin (67 kDa), Ovalbumin (43 kDa), Chymotrypsinogen $(25 \mathrm{kDa})$, and Ribonuclease $(17.3 \mathrm{kDa})$.

For the SEC-TDA, samples were separated according to their hydrodynamic size on the same Superdex 200 column in $25 \mathrm{mM}$ Tris- $\mathrm{HCl}, \mathrm{pH} 7.5,150 \mathrm{mM} \mathrm{KCl}$, and $1 \mathrm{mM} \beta$-mercaptoethanol at $20^{\circ} \mathrm{C}$. Molecular masses of the eluted sample were determined online on a TDA model 302 (Malvern Instruments Ltd, UK) at $20^{\circ} \mathrm{C}$. The TDA contains 4 detectors in line: (i) a static light-scattering cell with two photodiode detectors at $7^{\circ}$ for lowangle light scattering (LALS) and at $90^{\circ}$ for RALS, (ii) a deflection refractometer, (iii) a photometer, and (iv) a differential viscometer. BSA was used for molecular mass calibration, and all data were acquired and processed using the Omnisec software (Viscotek Ltd.) as described elsewhere [32].

\section{Coproduction of ClpP1 and ClpP2 with the ClpC and ClpX ATPases}

The $\operatorname{clpX}$ ORF (Rv2457c) was PCR amplified using the ClpX-5Nco and ClpX-3Hind primers and introduced at the NcoI and HindIII sites into the pCDFDuet vector (Novagen). The resulting plasmid was named pNB104. The $\operatorname{clpP1}$ ORF was amplified using the ClpP1-5Nde and ClpP1-3Pac primers and introduced at the NdeI and PacI in the pNB104 plasmid. The final plasmid (pNB106) contained the $\operatorname{clp} X$ and $\operatorname{clpP} 1$ (his) 6 under the control of two independent $\mathrm{T} 7$ promotors.

In order to express $c l p P 2$ together with $C l p X$, the $\operatorname{clpP} 2$ ORF was first amplified with the ClpP2-5Nco and ClpP2$\mathrm{CHis}$ primers and cloned into the pCDFDuet vector at the NcoI and HindIII sites. The resulting plasmid was named pNB79. The $c l p X$ ORF was amplified with the ClpX-5Nde and ClpX-3Pac primers and introduced into the pNB79 plasmid at the NdeI and PacI sites. The resulting plasmid (pNB107) contained the $c l p P 2($ his) 6 and the $c l p X$ ORF under two independent $\mathrm{T} 7$ promotors.

The $c l p C$ ORF (Rv3596c) was PCR amplified using the $\mathrm{ClpC}-5 \mathrm{Nco}$ and $\mathrm{ClpC}-3$ Hind primers and introduced at the NcoI and HindIII sites into the pCDFDuet vector. 
The resulting plasmid was named pNB108. Then, the clpP1 ORF introduced at the NdeI and PacI in the pNB108 plasmid as described for the pNB106 plasmid. The final plasmid (pNB110) contained the $\operatorname{clpC}$ and clpP1(his) ${ }_{6}$ under the control of two independent $\mathrm{T} 7$ promotors.

In order to express $c l p P 2$ together with $\operatorname{clp} C$, the $\operatorname{clpC}$ ORF was first amplified with the $\mathrm{ClpC}-5 \mathrm{Nde}$ and $\mathrm{ClpC}$ Pac primers and cloned into the pNB79 plasmid at the NdeI and PacI sites. The resulting plasmid was named pNB111 and it contained the $\operatorname{clpP} 2$ (his) ${ }_{6}$ and the $c l p X$ ORF under two independent T7 promotors.

SG1146a cells were transformed with the pNB104, pNB106, pNB107, pNB108, pNB110, pNB111 plasmids with or without pNB82 as indicated in the legends. Cells were cultivated at $30^{\circ} \mathrm{C}$ in LB medium complemented when necessary with $30 \mu \mathrm{g} / \mathrm{mL}$ of kanamycin, $50 \mu \mathrm{g} / \mathrm{mL}$ of streptomycin and $25 \mu \mathrm{g} / \mathrm{mL}$ of chloramphenicol. Protein production was induced with IPTG as described above and cells were harvested. Cells pellets were resuspended in $50 \mathrm{mM}$ Tris- $\mathrm{HCl} \mathrm{pH} \mathrm{7.5,} 200 \mathrm{mM} \mathrm{KCl}, 2$ mM DTT, $0.1 \mathrm{mM}$ EDTA, 10\% glycerol and lyzed by sonication. Peptide hydrolysis was measured in $50 \mathrm{mM}$ Tris $\mathrm{pH} 7.5,100 \mathrm{mM} \mathrm{KCl}$ and $1 \mathrm{mM} \mathrm{DTT}, 10 \mathrm{mM}$ $\mathrm{MgCl}_{2}, 4 \mathrm{mM}$ ATP and $0.02 \%$ Triton X100 as described above.

\section{Additional material}

Additional file 1: Figure S1: Sequence alignment of MTB ClpP1 and ClpP2 with that of $E$. coli ClpP. The sequences of MTB ClpP1 (H37Rv strain, gi: 41353667) and ClpP2 (H37Rv strain, gi: 2791500) were aligned with that of E. coli ClpP (gi: 89107307) using ClustalW program http:// www.ebi.ac.uk/Tools/msa/clustalw2. The propeptide of E. coli ClpP is written in bold. The residues in the catalytic triad (Ser, His, Asp) are indicated in bold and underlined. Identical $\left({ }^{*}\right)$, the similar (.), and very similar (:) residues are indicated below the sequences.

Additional file 2: Figure S2: Peptidase activity of E. coli ClpP copurified with ClpP1 and ClpP2. Hydrolysis of $1 \mathrm{mM}$ Suc-LY-Amc peptide in the presence of $10 \mu \mathrm{g}$ of $\mathrm{Ni}^{2+}$ column-purified proteins from BL21(DE3) cells overexpressing the pET26b plasmid (black triangles), BL21

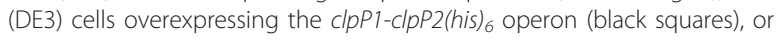
SG1146a cells overexpressing the CIPP1-clpP2(his) $)_{6}$ operon (black circles) after 50 days of storage of the protein preparation at $4^{\circ} \mathrm{C}$. Hydrolysis of the peptide was followed by measuring the release of amc (7-amino-4methylcoumarin) in a spectrofluorometer ( $\left.\lambda_{\text {ex }} 380 \mathrm{~nm} ; \lambda_{\mathrm{em}} 460 \mathrm{~nm}\right)$.

Additional file 3: Figure S3: Interaction of E. coli ClpP with ClpP1 or ClpP2. Soluble extracts were prepared from SG1146a cells producing untagged $E$. coli $\mathrm{ClpP}$ alone or together with $\mathrm{ClpP} 1(\mathrm{His})_{6}$ or $\mathrm{ClpP} 2(\mathrm{His})_{6}$ and loaded on a $\mathrm{Ni}^{2+}$ column. After extensive washing with buffer $\mathrm{A}$ (50 $\mathrm{mM} \mathrm{NaH} 2 \mathrm{PO} 4 \mathrm{pH}$ 8.0, $300 \mathrm{mM} \mathrm{NaCl}, 10 \mathrm{mM}$ Imidazole, $10 \%$ glycerol), resin-bound proteins were eluted with buffer $\mathrm{B}\left(50 \mathrm{mM} \mathrm{NaH}{ }_{2} \mathrm{PO} 4 \mathrm{pH}\right.$ 8.0, $300 \mathrm{mM} \mathrm{NaCl}, 250 \mathrm{mM}$ Imidazole, 10\% glycerol). The presence of $E$. coli ClpP was analyzed by a 15\% SDS-PAGE and detected by immunoblot using an anti ClpP antibody that exhibited cross-reaction with ClpP1 and CIPP2. (A) The indicated samples were loaded on a 15\% SDS-PAGE stained with Coomassie blue. The molecular mass markers are indicated on the left. Lanes 1-3: $10 \mu \mathrm{g}$ of the soluble extract of SG1146a cells producing untagged E. coli ClpP (lane 1), ClpP1(His)6 (lane 2), or untagged E. coli ClpP together with ClpP1(His)6 (lane 3) that were loaded on the $\mathrm{Ni}^{2+}$ column. Lanes 4-6: proteins eluted from $\mathrm{Ni}^{2+}$ column when SG1146a cells produced untagged E. coli ClpP (lane 4), ClpP1(His) 6 (lane 5), or untagged E. coli ClpP together with $\mathrm{ClpP} 1(\mathrm{His})_{6}$ (lane 6). The upper band in lane 6 is $\mathrm{ClpP} 1(\mathrm{His})_{6}$ as determined by anti His tag immunoblot (data not shown) and the lower band is E. coli ClpP as determined by anti ClpP immunodetection in the panel (C). (B) The indicated samples were loaded on a 15\% SDS-PAGE stained with Coomassie blue. The molecular mass markers are indicated on the left. Lanes 1-3: $10 \mu \mathrm{g}$ of the soluble extract of SG1146a cells producing untagged E. coli ClpP (lane 1), ClpP2(His) 6 (lane 2), or untagged E. coli ClpP together with ClpP2(His) 6 (lane 3) that were loaded on the $\mathrm{Ni}^{2+}$ column. Lanes 4-6: proteins eluted from $\mathrm{Ni}^{2+}$ column when SG1146a cells produced untagged E. coli ClpP (lane 4), ClpP2(His)6 (lane 5), or untagged E. coli ClpP together with $\mathrm{ClpP} 2(\mathrm{His})_{6}\left(\right.$ lane 6). The upper band in lane 6 is $\mathrm{ClpP} 2(\mathrm{His})_{6}$ as determined by anti His tag immunoblot (data not shown) and the lower band is E. coli ClpP as determined by anti ClpP immunodetection in the panel (C). (C) Immunodetection of ClpP proteins in $2 \mu \mathrm{g}$ of soluble extract of SG1146a cells producing E. coli ClpP (lane 1) and in the sample eluted from the $\mathrm{Ni}^{2+}$ column when the SG1146a cells produced untagged E. coli ClpP (lane 2), ClpP1(His) 6 (lane 3), ClpP2(His) 6 (lane 4), untagged $E$. coli ClpP together with $\mathrm{ClpP1}(\mathrm{His})_{6}$ (lane 5), or untagged $E$. coli ClpP together with ClpP2(His) 6 (lane 6). The proteins were separated on a $15 \%$ SDS-PAGE and transferred onto nitrocellulose. The ClpP proteins were detected using an anti ClpP antibody that interacted with MTB ClpP1 and ClpP2 as well as with E. coli ClpP. Experimental evidence of an interaction between E. coli ClpP and MTB ClpP1 or ClpP2 was also observed by producing E. coli ClpP(His) 6 together with S-tagged ClpP1 or ClpP2 (data not shown).

Additional file 4: Figure S4: Purified ClpP1 and ClpP2 variants. About $5 \mu \mathrm{g}$ of the indicated purified proteins were loaded on a $12 \%$ SDS-PAGE stained with Coomassie blue. The molecular mass markers are indicated on the left. (A) Full length ClpP1 (M1) and the variants starting at the Met7 (M7) and Ser9 (S9). (B) Full length ClpP2 (M1) and the variants starting at Arg13 (R13), Tyr14 (Y14), lle15 (115), Leu16 (L16). (C) Full length ClpP1 (M1) and the variants starting at the Ser11 (S11), Gln12 (Q12), Leu16 (L16), and Ser19 (S19). (D) Full length ClpP2 (M1) and the variants starting at the Ser23 (S23), Ser24 (S24), Lys28 (K28), and Asn31 (N31).

Additional file 5: Table S1: Oligonucleotides used in this study.

\section{List of abbreviation used}

Clp: caseinolytic protease; ORF: open reading frame; MTB: Mycobacterium tuberculosis; Suc-LY-Amc: N-Succinyl-Leu-Tyr-7 amido 4 methylcoumarin; BzVGR-Amc: benzoyl-Val-Gly-Arg-7 amido 4 methylcoumarin; Boc-LRR-Amc: terbutyloxycarbonyl-Leu-Arg-Arg-7 amido 4 methylcoumarin; SEC: sizeexclusion chromatography; SEC-TDA: size-exclusion chromatography coupled online to a triple detector array; DTT: dithiothreitol; ATP: adenosine triphosphate; IPTG: isopropyl- $\beta$-D-thiogalactopyranoside.

\section{Acknowledgements}

The authors thank J. D'Alayer (Institut Pasteur) for microsequencing Ntermini of ClpP1 and ClpP2 and S. Gottesman (National institute of Health) for the SG1146a strain. The authors are very grateful to C. Wandersman (Institut Pasteur) and G. Karimova (Institut Pasteur) for critically reading the manuscript and to A. Chenal (Institut Pasteur) for fruitful discussions on the SEC-TDA. The authors are indebted to M. Picardeau (Institut Pasteur) for his constant support for the completion of this project.

\section{Author details}

'Institut Pasteur, Unité de Biologie des Spirochètes, Institut Pasteur, F-75015 Paris France. ${ }^{2}$ Institut Pasteur, Plate-Forme de Biophysique des Macromolécules et de leurs Interactions, Institut Pasteur, F-75015 Paris France. ${ }^{3}$ Laboratory of Molecular Biology, National Cancer Institute, National Institutes of Health, Bethesda, MD 20892, USA. ${ }^{4}$ Architecture et Fonction des Macromolécules Biologiques (UMR6098), CNRS, Universités d'Aix-Marseille I \& II, 163 Avenue de Luminy, 13288 Marseille cedex 9, France. 


\section{Authors' contributions}

NB conceived, designed and performed experiments, analyzed data and wrote the manuscript. BR carried out and analyzed Sec-TDA experiments. MM helped in purifying and characterizing ClpP1 and ClpP2. MO-L helped in purifying processed ClpP1 and ClpP2. All read and approved the final manuscript.

Received: 8 July 2011 Accepted: 1 December 2011

Published: 1 December 2011

\section{References}

1. Gottesman S: Proteolysis in bacterial regulatory circuits. Annu Rev Cell Dev Biol 2003, 19:565-587.

2. Goldberg AL: Protein degradation and protection against misfolded or damaged proteins. Nature 2003, 426(6968):895-899.

3. Wang J, Hartling JA, Flanagan JM: The structure of ClpP at $2.3 \mathrm{~A}$ resolution suggests a model for ATP-dependent proteolysis. Cell 1997, 91(4):447-456.

4. Thompson MW, Maurizi MR: Activity and specificity of Escherichia coli ClpAP protease in cleaving model peptide substrates. J Biol Chem 1994, 269(27):18201-18208.

5. Woo KM, Chung WJ, Ha DB, Goldberg AL, Chung CH: Protease Ti from Escherichia coli requires ATP hydrolysis for protein breakdown but not for hydrolysis of small peptides. J Biol Chem 1989, 264(4):2088-2091.

6. Kress W, Maglica Z, Weber-Ban E: Clp chaperone-proteases: structure and function. Res Microbiol 2009, 160(9):618-628.

7. Frieden TR, Sterling TR, Munsiff SS, Watt CJ, Dye C: Tuberculosis. Lancet 2003, 362(9387):887-899

8. Parrish NM, Dick JD, Bishai WR: Mechanisms of latency in Mycobacterium tuberculosis. Trends Microbiol 1998, 6(3):107-112.

9. Amer AO, Swanson MS: A phagosome of one's own: a microbial guide to life in the macrophage. Curr Opin Microbiol 2002, 5(1):56-61.

10. Schnappinger D, Ehrt S, Voskuil MI, Liu Y, Mangan JA, Monahan IM, Dolganov G, Efron B, Butcher PD, Nathan C, et al: Transcriptional Adaptation of Mycobacterium tuberculosis within Macrophages: Insights into the Phagosomal Environment. J Exp Med 2003, 198(5):693-704.

11. Webb C, Moreno M, Wilmes-Riesenberg M, Curtiss R, Foster JW: Effects of DksA and ClpP protease on sigma $S$ production and virulence in Salmonella typhimurium. Mol Microbiol 1999, 34(1):112-123.

12. Gaillot O, Pellegrini E, Bregenholt S, Nair S, Berche P: The ClpP serine protease is essential for the intracellular parasitism and virulence of Listeria monocytogenes. Mol Microbiol 2000, 35(6):1286-1294.

13. Robertson GT, Ng WL, Foley J, Gilmour R, Winkler ME: Global transcriptional analysis of clpP mutations of type 2 Streptococcus pneumoniae and their effects on physiology and virulence. J Bacteriol 2002, 184(13):3508-3520.

14. Frees D, Qazi SN, Hill PJ, Ingmer H: Alternative roles of ClpX and ClpP in Staphylococcus aureus stress tolerance and virulence. Mol Microbiol 2003, 48(6):1565-1578

15. Loughlin MF, Arandhara V, Okolie C, Aldsworth TG, Jenks PJ: Helicobacter pylori mutants defective in the clpP ATP-dependant protease and the chaperone clpA display reduced macrophage and murine survival. Microb Pathog 2009, 46(1):53-57.

16. Sherrid AM, Rustad TR, Cangelosi GA, Sherman DR: Characterization of a Clp protease gene regulator and the reaeration response in Mycobacterium tuberculosis. PLoS One 2010, 5(7):e11622.

17. Sassetti CM, Boyd DH, Rubin EJ: Genes required for mycobacterial growth defined by high density mutagenesis. Mol Microbiol 2003, 48(1):77-84.

18. Estorninho M, Smith $H$, Thole J, Harders-Westerveen J, Kierzek A, Butler RE, Neyrolles O, Stewart GR: ClgR regulation of chaperone and protease systems is essential for Mycobacterium tuberculosis parasitism of the macrophage. Microbiology 2010, 156(Pt 11):3445-3455.

19. Maurizi MR, Clark WP, Katayama Y, Rudikoff S, Pumphrey J, Bowers B, Gottesman S: Sequence and structure of Clp P, the proteolytic component of the ATP-dependent Clp protease of Escherichia coli. J Biol Chem 1990, 265(21):12536-12545.

20. Maurizi MR, Clark WP, Kim SH, Gottesman S: Clp P represents a unique family of serine proteases. J Biol Chem 1990, 265(21):12546-12552.

21. Ingvarsson H, Mate MJ, Hogbom M, Portnoi D, Benaroudj N, Alzari PM, Ortiz-Lombardia M, Unge T: Insights into the inter-ring plasticity of caseinolytic proteases from the X-ray structure of Mycobacterium tuberculosis ClpP1. Acta Crystallogr D Biol Crystallogr 2007, 63(Pt 2):249-259.

22. Viala J, Mazodier P: ClpP-dependent degradation of PopR allows tightly regulated expression of the clpP3 clpP4 operon in Streptomyces lividans. Mol Microbiol 2002, 44(3):633-643.

23. Bewley MC, Graziano V, Griffin K, Flanagan JM: The asymmetry in the mature amino-terminus of ClpP facilitates a local symmetry match in ClpAP and ClpXP complexes. J Struct Biol 2006, 153(2):113-128.

24. Bewley MC, Graziano V, Griffin K, Flanagan JM: Turned on for degradation: ATPase-independent degradation by ClpP. J Struct Biol 2009, 165(2):118-125.

25. Kohler A, Cascio P, Leggett DS, Woo KM, Goldberg AL, Finley D: The axial channel of the proteasome core particle is gated by the Rpt2 ATPase and controls both substrate entry and product release. Mol Cell 2001, 7(6):1143-1152.

26. Kang SG, Dimitrova MN, Ortega J, Ginsburg A, Maurizi MR: Human mitochondrial ClpP is a stable heptamer that assembles into a tetradecamer in the presence of ClpX. J Biol Chem 2005, 280(42):35424-35432.

27. Kimber MS, Yu AY, Borg M, Leung E, Chan HS, Houry WA: Structural and theoretical studies indicate that the cylindrical protease ClpP samples extended and compact conformations. Structure 2010, 18(7):798-808

28. Arribas J, Castano JG: A comparative study of the chymotrypsin-like activity of the rat liver multicatalytic proteinase and the ClpP from Escherichia coli. J Biol Chem 1993, 268(28):21165-21171.

29. Lin W, Chan M, Sim TS: Atypical caseinolytic protease homolog from Plasmodium falciparum possesses unusual substrate preference and a functional nuclear localization signal. Parasitol Res 2009, 105(6):1715-1722.

30. Thompson MW, Miller J, Maurizi MR, Kempner E: Importance of heptameric ring integrity for activity of Escherichia coli ClpP. Eur J Biochem 1998, 258(3):923-928.

31. Studier FW, Rosenberg AH, Dunn JJ, Dubendorff JW: Use of T7 RNA polymerase to direct expression of cloned genes. Methods Enzymol 1990, 185:60-89

32. Sotomayor Perez AC, Karst JC, Davi M, Guijarro JI, Ladant D, Chenal A: Characterization of the regions involved in the calcium-induced folding of the intrinsically disordered RTX motifs from the Bordetella pertussis adenylate cyclase toxin. J Mol Biol 2010, 397(2):534-549.

doi:10.1186/1471-2091-12-61

Cite this article as: Benaroudj et al:: Assembly and proteolytic processing of mycobacterial ClpP1 and ClpP2. BMC Biochemistry 2011 12:61.

\section{Submit your next manuscript to BioMed Central and take full advantage of:}

- Convenient online submission

- Thorough peer review

- No space constraints or color figure charges

- Immediate publication on acceptance

- Inclusion in PubMed, CAS, Scopus and Google Scholar

- Research which is freely available for redistribution 